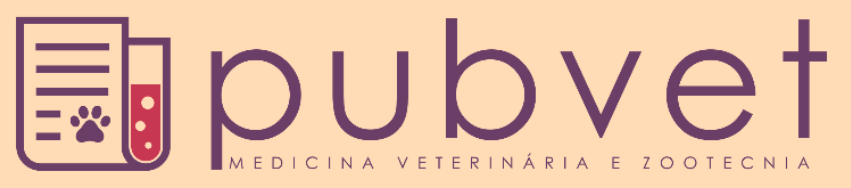

https://doi.org/10.31533/pubvet.v13n11a453.1-7

\title{
Morfometria geométrica das asas permite verificar o posicionamento racial de abelhas africanizadas
}

Fábio Adriano Santos e Silva ${ }^{1 \bullet}$, Darcet Costa Souza ${ }^{2} \bullet$, Arnaud Azevedo Alves ${ }^{2}{ }^{\circ}$, José Elivalto Guimarães Campelo ${ }^{\circ}{ }^{\circ}$, Juliana do Nascimento Bendini ${ }^{3}{ }^{\circ}$, Lorena Andrade Nunes $^{4}{ }^{\circ}$, Jaqueline Rosemeire Verzignassi ${ }^{\circ}{ }^{\circ}$, Francielly Paludo ${ }^{6}{ }^{\circ}$, Patrick Bezerra Fernandes $^{7^{*}}$, Jackson Rocklley Gomes da Silva ${ }^{8}$, Josélia Quaresma da Silva $^{9} \bullet$

${ }^{1}$ Engenheiro agrônomo, mestre em Ciência Animal.

${ }^{2}$ Engenheiro agrônomo, doutor, professor - Departamento de Zootecnia - CCA - Universidade Federal do Piauí - UFPI.

${ }^{3}$ Bióloga, doutora, professora - Universidade Federal do Piauí UFPI.

${ }^{4}$ Bióloga, doutora em Entomologia, professora da UESB.

${ }^{5}$ Engenheira agrônoma, doutora, pesquisadora - Empresa Brasileira de Pesquisa Agropecuária-Embrapa Gado de Corte.

${ }^{6}$ Médica veterinária, mestranda em Zootecnia - IF goiano - Campus Rio Verde.

${ }^{7}$ Zootecnista, mestre em Zootecnia.

${ }^{8}$ Aluno do curso de zootecnia da Universidade Estadual de Goiás.

${ }^{9}$ Bióloga, especialista em Gestão de Recursos Humanos, assistente em Administração do IFPI.

*Autor para correspondência, E-mail: zoo.patrick@hotmail.com

Resumo. Este trabalho teve como objetivo verificar as possíveis diferenças entre populações de abelhas africanizadas da região litorânea e do sertão do Piauí, utilizando como técnica a morfometria geométrica. O material analisado foi constituído de operárias adultas de abelhas africanizadas provenientes da região litorânea e do sertão do Piauí. Utilizaram-se como referência comparativa de raças as imagens digitais das asas de 50 indivíduos das subespécies europeias, africana e 45 indivíduos de abelhas africanizadas oriundas de Ribeirão Preto nas análises morfométricas. No total foram analisadas 645 imagens de asas. Na análise morfométrica, foram utilizados os padrões de venações da asa anterior direita com 19 landmarks. Para a realização dos estudos de morfometria geométrica, as peças foram registradas e analisadas com o auxílio dos programas tpsDig e MorphoJ. Nas análises das variáveis canônicas, foram necessárias as três primeiras variáveis para acumular $86 \%$ da variação total observada. Houve diferenças significativas $(\mathrm{P}<0,001)$ na análise de multivariada entre os 7 grupos estudados. As distâncias quadradas de Mahalanobis se mostraram estatisticamente significante. Através da morfometria geométrica das asas foi possível diferenciar as subespécies avaliadas e se verificar o posicionamento racial das abelhas africanizadas do Piauí. Não fica evidente a existência de ecótipos entre as abelhas da região litorânea e sertão piauiense.

Palavras chave: Apis melífera, multivariada, variável canônica

\section{Geometric morphometry of the wings allows verifying the racial positioning of Africanized bees}

Abstract. This study aimed to determine possible differences between populations of Africanized bees in the coastal area and of the sertão of Piauí, as a technique using geometric morphometry. The material analyzed consisted of adult workers of Africanized bees from the coastal region and of the sertão of Piauí. It was used as a comparative reference breeds digital images of the wings of 50 individual's subspecies of European, African, and 45 individuals of Africanized bees coming from Ribeirao Preto in morphometric analyzes. In total we analyzed 645 images of wings. In the morphometric 
analysis, we used the standards veined right forewing with 19 landmarks. For the studies of geometric morphometric, the pieces were recorded and analyzed with the aid of programs and tpsDig MorphoJ. In the analysis of canonical variables, were the first three variables necessary to accumulate $86 \%$ of the total variation. There were significant differences $(\mathrm{P}<0.001)$ in multivariate analysis among the seven groups. The squared Mahalanobis distances were statistically significant. By geometric morphometrics of the wings could differentiate the subspecies evaluated and check the positioning of racial Africanized bees Piauí. There is evident the existence of ecotypes among bees littoral and hinterland Piauí.

Keywords: Apis melífera, multivariate, canonical variable

\section{Morfometría geométrica de las alas permite verificar el posicionamiento racial de las abejas africanizadas}

Resumen. Este estudio tenía como objetivo verificar las posibles diferencias entre las poblaciones de abejas africanizadas de la región costera y el interior de Piauí, utilizando como técnica la morfometría geométrica. El material analizado fue consistuido de obreras adultas de abejas africanizadas de la región costera y el interior de Piauí. Fueron utilizadas como referencia comparativa de razas las imágenes digitales de las alas de 50 individuos de la subespecie europea africana y 45 individuos de abejas africanizadas originarias de Ribeirão Preto en los análisis morfométricos. En total, se analizaron 645 imágenes de alas. En el análisis morfométrico, los patrones de venación del ala anterior derecha se utilizaron con 19 puntos de referencia. Para realizar los estudios geométricos de morfometría, las piezas fueron grabadas y analizadas con la ayuda de los programas tpsDig y MorphoJ. En los análisis de las variables canónicas, las tres primeras variables fueron necesarias para acumular el $86 \%$ de la variación total observada. Hubo diferencias significativas ( $\mathrm{P}<$ 0.001) en el análisis multivariado entre los 7 grupos estudiados. Las distancias cuadradas de Mahalanobis mostraron ser estadísticamente significativas. A través de la morfometría geométrica de las alas fue posible diferenciar la subespecie evaluada y verificar el posicionamiento racial de las abejas africanizadas de Piauí. No es evidente la existencia de ecotipos entre las abejas de la región costera y el interior de Piauiense.

Palabras clave: Apis melífera, multivariada, variable canónica

\section{Introdução}

A Apis mellifera é encontrada em todas as partes do mundo, com exceção das regiões polares, enfrentando condições ecológicas bastante diversificadas que incluem savanas, florestas de clima tropical, desertos, regiões litorâneas e montanhosas (Arruda et al., 2007; Quiroga, 2004). Essa grande variedade de clima e vegetação acabou originando uma resposta adaptativa às diversas subespécies de abelhas, com diferentes características e adaptadas às diversas condições ambientais.

No estado do Piauí, as abelhas africanizadas são encontradas em diversos ecossistemas (caatinga, cerrado, mata de babaçu, ecótonos e vegetação do litoral), o que indica sua adaptabilidade as condições naturais do estado. Os estudos morfométricos são muito utilizados sobre a estrutura populacional e sobre a variação geográfica de subespécies ou entre populações de abelhas, principalmente em Apis mellifera (Nunes et al., 2007). A morfometria é uma ferramenta importante para a definição e separação de espécies, subespécies e ecótipos (Francoy \& Imperatriz-Fonseca, 2010).

O conhecimento dos padrões raciais é uma informação importante tanto para ajustes e adequações de tecnologias, quanto para o entendimento da adaptação das populações de abelhas africanizadas. Conhecer a maior ou menor proximidade das populações atuais das subespécies europeias e/ou africanas ajudará a entender melhor a biologia dessas abelhas.

Diante disso, foram verificadas por meio de análise morfométrica as diferenças entre populações de abelhas africanizadas e a possível existência de ecótipos da região litorânea e do sertão do Piauí. 


\section{Material e métodos}

O material analisado foi constituído de operárias adultas de abelhas africanizadas provenientes da região litorânea e do sertão do Piauí. As coletas das amostras foram realizadas no período de dezembro de 2012 a maio de 2013. As abelhas coletadas foram retiradas da parte central da colmeia, com o objetivo de ter abelhas operárias jovens, para que não houvesse danos nas nervuras da asa anterior direita. Em cada região foram coletadas amostras de 10 apiários, sendo coletadas 10 operárias por colônia e duas colônias por apiário. No sertão foi escolhido o município de São Raimundo Nonato ( $9^{\circ} 0^{\prime} 54^{\prime \prime} \mathrm{S}, 42^{\circ} 41^{\prime}$ $\left.56^{\prime \prime} \mathrm{W}\right)$ e no litoral foi feita nos municípios de Parnaíba ( $\left.2^{\circ} 54^{\prime} 18^{\prime \prime} \mathrm{S}, 41^{\circ} 46^{\prime} 37^{\prime \prime} \mathrm{W}\right)$ e Buriti dos Lopes $\left(3^{\circ} 10^{\prime} 30^{\prime \prime} \mathrm{S}, 41^{\circ} 52^{\prime} 1^{\prime \prime} \mathrm{W}\right)$ que se destacam na apicultura (Figura 1$)$.
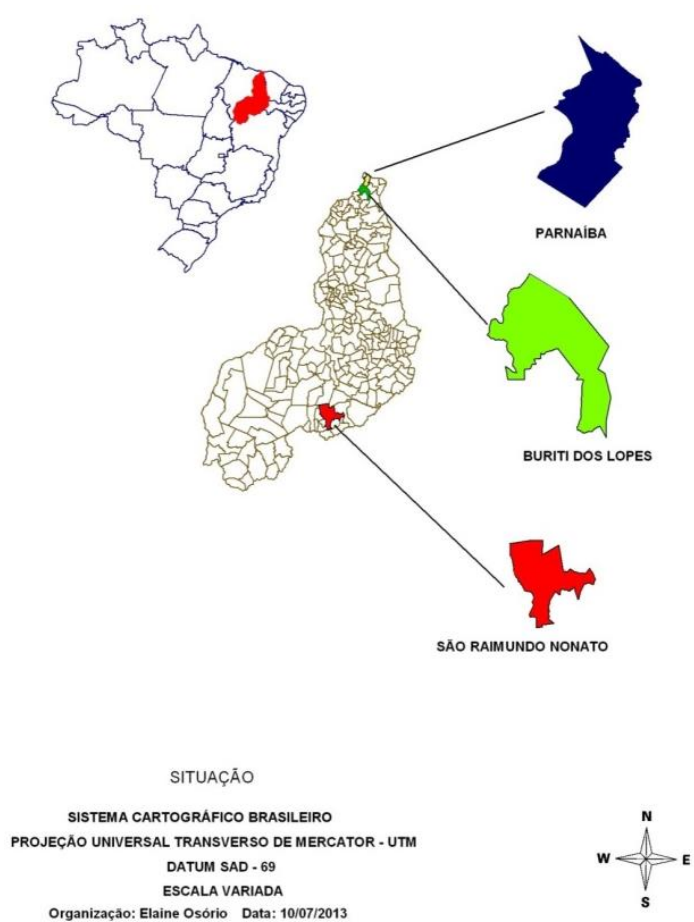

Figura 1. Mapa indicando os municípios de coleta das amostras de abelhas.

Os apiários amostrados foram georeferenciados (Tabela 1). As abelhas foram coletadas em apiários fixos e conservadas em álcool $70 \%$ para posterior análise. As amostras foram devidamente identificadas com o local de coleta e número da amostra.

Para análise morfométricas, foram utilizados os padrões de venação das asas anteriores das subespécies Apis mellifera ligustica, Apis mellifera carnica, Apis mellifera melífera e Apis mellifera scutellata que foram obtidas a partir de imagens digitais. Estas imagens foram utilizadas nas análises morfométricas como base de comparação e verificação da influência de cada subespécie no perfil morfométrico das populações de abelhas africanizadas avaliadas nesta pesquisa. Assim, foram utilizadas imagens digitais das asas de 50 indivíduos de cada subespécie e 45 de abelhas africanizadas oriundas de Ribeirão Preto, nas análises morfométricas, que associadas às das populações em análise, perfizeram 645 amostras. As análises de morfometria geométrica foram realizadas no laboratório do Setor de Apicultura do Departamento de Zootecnia da Universidade Federal do Piauí em Teresina, Piauí.

Foram utilizadas asas anteriores direitas de abelhas operárias, retiradas com o auxílio de tesoura e montadas entre lâmina e lamínula. As imagens das asas foram capturadas com o software Motic 2.0 ML utilizando-se câmera digital acoplada a um estereomicroscópio e armazenadas em computador. As imagens capturadas foram separadas por localidade e região. Após a preparação das lâminas, as abelhas foram identificadas e colocadas em tubos tipo Eppendorf com capacidade de 2,0 mL contendo álcool $70 \%$, e preservados individualmente.

A morfometria das asas foi analisada com o auxílio do software tpsDig (Rohlf, 2005). Foram colocados marcos anatômicos nas junções das nervuras das células das asas e foi realizada análise de 
deformações parciais e relativas utilizando-se o software MorphoJ. Foram ainda realizadas análises de variáveis canônicas e análises discriminantes com os dados de deformações parciais e relativas para a verificação da variabilidade e do poder de discriminação da técnica dos grupos avaliados.

Tabela 1. Municípios, coordenadas, altitude e número de amostras de abelhas africanizadas utilizados no estudo.

\begin{tabular}{|c|c|c|c|c|}
\hline \multirow{2}{*}{ Municípios } & \multicolumn{2}{|c|}{ Coordenadas geográficas } & \multirow{2}{*}{ Altitude (m) } & \multirow{2}{*}{ Número de Amostras } \\
\hline & Latitude & Longitude & & \\
\hline Buriti dos Lopes & $-03^{\circ} 20^{\prime} 01,30^{\prime \prime} \mathrm{S}$ & $-42^{\circ} 01^{\prime} 09,50^{\prime \prime} \mathrm{W}$ & 74 & 20 \\
\hline Buriti dos Lopes & $-03^{\circ} 20^{\prime} 01,11^{\prime \prime} \mathrm{S}$ & $-42^{\circ} 01^{\prime} 09,46^{\prime \prime} \mathrm{W}$ & 80 & 20 \\
\hline Parnaiba & $-03^{\circ} 03^{\prime} 38,36^{\prime \prime} \mathrm{S}$ & $-41^{\circ} 43^{\prime} 38,72^{\prime \prime} \mathrm{W}$ & 71 & 20 \\
\hline Parnaiba & $-03^{\circ} 02^{\prime} 17,93^{\prime \prime S}$ & $-41^{\circ} 43^{\prime} 30,06^{\prime \prime} \mathrm{W}$ & 65 & 20 \\
\hline Parnaiba & $-03^{\circ} 03^{\prime} 57,92^{\prime \prime} \mathrm{S}$ & $-41^{\circ} 41^{\prime} 35,51^{\prime \prime} \mathrm{W}$ & 90 & 20 \\
\hline Parnaiba & $-03^{\circ} 03^{\prime} 54,53^{\prime \prime} \mathrm{S}$ & $-41^{\circ} 44^{\prime} 57,53^{\prime \prime} \mathrm{W}$ & 46 & 20 \\
\hline Parnaiba & $-03^{\circ} 03^{\prime} 51,07^{\prime} \mathrm{S}$ & $-41^{\circ} 44^{\prime} 50,86^{\prime \prime} \mathrm{W}$ & 49 & 20 \\
\hline Parnaiba & $-03^{\circ} 02^{\prime} 40,26^{\prime \prime} \mathrm{S}$ & $-41^{\circ} 37^{\prime} 54,99^{\prime \prime} \mathrm{W}$ & 68 & 20 \\
\hline Parnaiba & $-03^{\circ} 03^{\prime} 54,53^{\prime \prime} \mathrm{S}$ & $-41^{\circ} 44^{\prime} 57,53^{\prime \prime} \mathrm{W}$ & 46 & 20 \\
\hline Parnaiba & $-03^{\circ} 03^{\prime} 51,07^{\prime \prime} \mathrm{S}$ & $-41^{\circ} 44^{\prime} 50,86^{\prime \prime} \mathrm{W}$ & 49 & 20 \\
\hline São Raimunto Nonato & $-08^{\circ} 49^{\prime} 45,47^{\prime \prime} \mathrm{S}$ & $-42^{\circ} 50^{\prime} 14,63^{\prime \prime} \mathrm{W}$ & 465 & 20 \\
\hline São Raimundo Nonato & $-09^{\circ} 00^{\prime} 43,09^{\prime} \mathrm{S}$ & $-42^{\circ} 47^{\prime} 40,96^{\prime \prime} \mathrm{W}$ & 403 & 20 \\
\hline São Raimundo Nonato & $-09^{\circ} 02^{\prime} 03,72^{\prime \prime S}$ & $-42^{\circ} 48^{\prime} 53,96^{\prime \prime} \mathrm{W}$ & 413 & 20 \\
\hline São Raimunro Nonato & $-09^{\circ} 02^{\prime} 08,23^{\prime \prime} \mathrm{S}$ & $-42^{\circ} 46^{\prime} 29,85^{\prime \prime} \mathrm{W}$ & 403 & 20 \\
\hline São Raimundo Nonato & $-08^{\circ} 49^{\prime} 45,58^{\prime \prime} \mathrm{S}$ & $-42^{\circ} 50^{\prime} 14,63^{\prime \prime} \mathrm{W}$ & 572 & 20 \\
\hline São Raimundo Nonato & $-08^{\circ} 49^{\prime} 45,47^{\prime \prime} \mathrm{S}$ & $-42^{\circ} 50^{\prime} 14,63^{\prime \prime} \mathrm{W}$ & 465 & 20 \\
\hline São Raimundo Nonato & $-09^{\circ} 11^{\prime} 41,17^{\prime} \mathrm{S}$ & $-42^{\circ} 43^{\prime} 41,14^{\prime \prime} \mathrm{W}$ & 478 & 20 \\
\hline São Raimundo Nonato & $-09^{\circ} 15^{\prime} 13,68^{\prime \prime} \mathrm{S}$ & $-42^{\circ} 41^{\prime} 41,90^{\prime \prime} \mathrm{W}$ & 445 & 20 \\
\hline São Raimundo Nonato & $-09^{\circ} 03^{\prime} 41,61^{\prime \prime} \mathrm{S}$ & $-42^{\circ} 56^{\prime} 48,24^{\prime \prime} \mathrm{W}$ & 459 & 20 \\
\hline São Raimundo Nonato & $-08^{\circ} 59^{\prime} 19,45^{\prime \prime} \mathrm{S}$ & $-42^{\circ} 43^{\prime} 26,07^{\prime \prime} \mathrm{W}$ & 361 & 20 \\
\hline Total & & & & 400 \\
\hline
\end{tabular}

Para avaliação da morfometria geométrica foram utilizados 19 marcos anatômicos (Figura 2) de cada amostra, conforme a metodologia de Francoy (2007), para serem tomada de medidas das asas anterior direita, visando relacionar o tamanho e a forma dessas estruturas. A ordem de introdução dos marcos anatômica foi a mesma para cada indivíduo, por ser este um requisito para estabelecer a homologia espacial das estruturas mediante suas coordenadas. Esses marcos anatômicos foram registrados utilizando-se o software TpsDig versão 2.04, disponível na internet e é de uso livre.

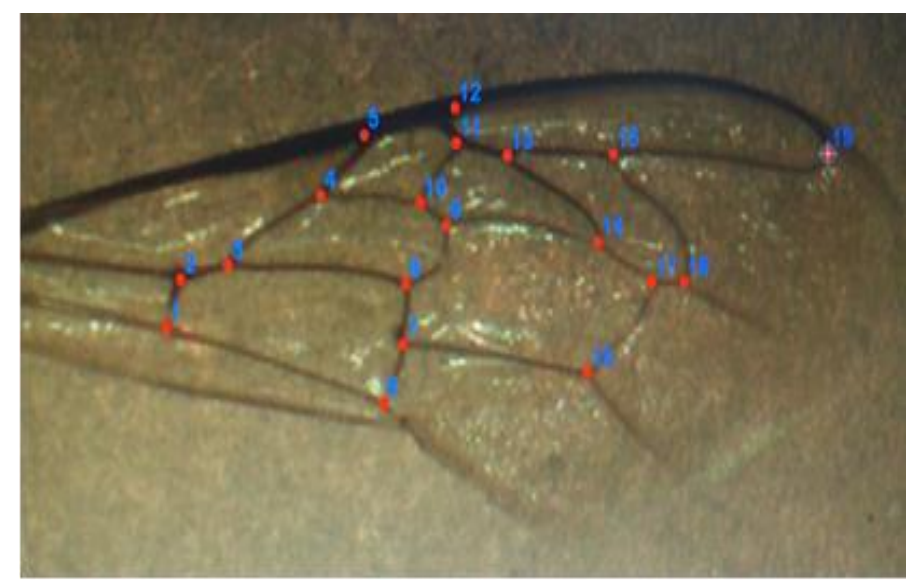

Figura 2. Asa anterior direita de uma abelha africanizada com os 19 marcos anatômicos em vermelho

Os dados foram submetidos à análise multivariada de variável canônica; assim foi possível estimar a primeira, segunda e terceira variável canônica. 


\section{Resultados e discussão}

$\mathrm{Na}$ análise das variáveis canônicas (AVC), foram necessárias as três primeiras variáveis para acumular cerca de $88 \%$ da variação total disponível, estando distribuídas da seguinte forma: $61,30 \%$ para a primeira, $16,66 \%$ para a segunda e $10,36 \%$ para a terceira variável canônica, ou seja, as duas primeiras variáveis canônicas foram suficientes para reunir mais de 77,95\% da variação total, viabilizando o estudo da divergência morfométrica entre as subespécies avaliadas (Tabela 2).

Tabela 2. Variáveis canônicas, autovalores, percentagem de variância e da variância acumulada obtidas com a análise de 6 características morfométricas das asas de Apis mellifera.

\begin{tabular}{lccc}
\hline Variável canônica & Autovalores & Proporção (\%) & Proporção Acumulada (\%) \\
\hline Can1 & 51,702 & 61,303 & 61,303 \\
Can2 & 14,054 & 16,658 & 77,961 \\
Can3 & 0,8761 & 10,358 & 88,346 \\
\hline
\end{tabular}

Pois, este estudo pode ser feito através da técnica de variáveis canônicas (Fonseca et al., 2000). Nunes et al. (2012), estudando a variação morfométrica das abelhas africanizadas (Apis mellifera) no Brasil, usando em suas análises as asas encontrou nas seis primeiras variáveis canônicas uma variação total de $76 \%$ da população em estudo. Esse resultado difere do encontrado nessa pesquisa, pois foram utilizadas amostras das subespécies europeias formadoras das atuais abelhas africanizadas. Podendo afirmar que a A. mellifera é uma espécie capaz de estabelecer de forma eficiente em diferentes regiões visando o seu rápido desenvolvimento e uma fácil adaptação (Nunes et al., 2012).

A partir das diferenças observadas nas AVC, foi plotado um gráfico de dispersão bidimensional, utilizando as duas primeiras variáveis canônicas a fim de constatar de forma mais clara as diferenças entre as populações e a formação de grupos distintos. Dessa forma, foi evidenciada a formação de três grupos distintos (Figura 3), um formado pelas subespécies A. Mellifera carnica e A. Mellifera ligustica. Esta aproximação pode ser pelo fato dessas subespécies terem a sua origem próximo uma da outra (Winston, 1991). Um segundo grupo formado pela sobreposição da A. mellifera mellifera, porém uma maior aproximação entre A. Mellifera scutellata e Ribeirão Preto. Essa aproximação deve-se ao fato do apiário de Ribeirão Preto ser um apiário experimental, onde constantemente são introduzidas matrizes de diversas linhagens e isso pode afetar de alguma maneira os padrões de asa das abelhas. E um terceiro grupo formado pela sobreposição dos indivíduos coletados no litoral e sertão do estado do Piauí. Essa sobreposição deve-se ao fato delas serem da mesma subespécie. Podemos ainda inferir destes dados (Figura 3) que as abelhas do Piauí são mais diferenciadas em relação a A. Mellifera scutellata do que as abelhas africanizadas de Ribeirão Preto.

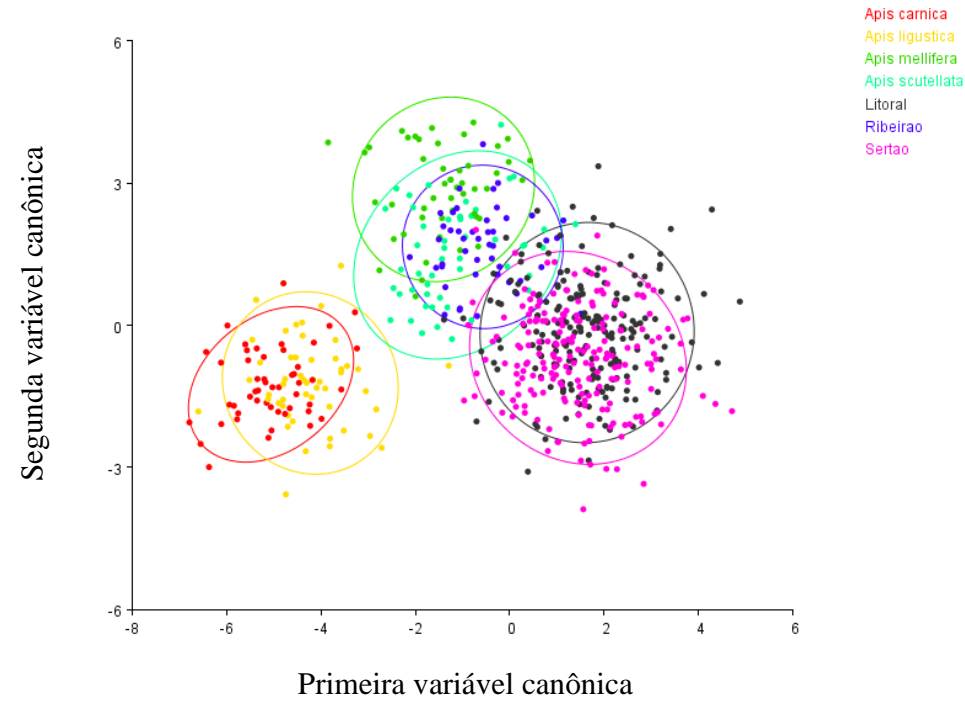

Figura 3. Análise das variáveis canônicas das populações de abelhas A. mellifera coletadas em Ribeirão Preto e nas regiões do litoral e sertão do Piauí juntamente com as subespécies formadoras das abelhas africanizadas. 
A pequena variação entre as abelhas africanizadas do litoral e sertão indica grande homogeneidade para o padrão de venação das asas anteriores, indicando não ter uma separação de ecótipos entre esses dois ecossistemas. Souza et al. (2009) estudando a morfometria de Apis Mellifera da mesorregião do sertão paraibano encontrou em seus resultados dois grupos distintos morfometricamente, o que se assemelha com esse estudo.

Os resultados encontrados nesse estudo (Figura 3) são semelhantes aos obtidos por Nunes (2012), que avaliando populações de A. mellifera, constataram que há uma grande variação entre as populações encontradas no Brasil e que isso é esperado pelo fato dessa espécie apresentar grande capacidade de se estabelecer eficientemente como populações silvestres em diferentes regiões, além de possuir características como rápido desenvolvimento e fácil adaptação e pela sua ampla distribuição propiciando assim a manutenção de um pool gênico.

As distâncias $\left(\mathrm{D}^{2}\right)$ de Mahalanobis entre os centróides das distribuições de cada grupo se mostraram estatisticamente significante (Tabela 3), ou seja, as populações estudadas apresentaram diferenças morfométricas a partir da forma das asas. Além disso, é possível verificar que a maior distância ocorreu entre a A. Mellifera carnica e o grupo do litoral do Piauí e a menor distância foi entre os grupos do litoral e sertão do Piauí. Esses resultados indicam que existe uma maior distância genética entre a $A$. mellifera carnica e o grupo do litoral do Piauí e a menor distância genética entre os grupos do litoral e sertão do Piauí, levando em consideração os caracteres morfológicos das asas utilizados nesse estudo.

Tabela 3. Distâncias quadradas de Mahalanobis entre as populações estudadas.

\begin{tabular}{lccccccc}
\hline & $A$, m, carnica & $A$, m, ligustica & $A$, m, mellifera & $A$, m, scutellata & Litoral & Ribeirão & Sertão \\
\hline A, m, carnica & & $<0,001$ & $<0,001$ & $<0,001$ & $<0,001$ & $<0,001$ & $<0,001$ \\
A, m, ligustica & 2,99 & & $<0,001$ & $<0,001$ & $<, 0,001$ & $<0,001$ & $<0,001$ \\
A, m, mellifera & 6,22 & 5,23 & & $<0,001$ & $<0,001$ & $<0,001$ & $<0,001$ \\
A, m, scutellata & 5,39 & 5,25 & 4,10 & & $<0,001$ & $<0,001$ & $<0,001$ \\
Litoral & 6,91 & 6,21 & 4,78 & 4,10 & & $<0,001$ & $<0,001$ \\
Ribeirão & 5,89 & 5,87 & 4,57 & 3,69 & 3,96 & & $<0,001$ \\
Sertão & 6,75 & 5,93 & 4,95 & 4,07 & 1,49 & 4,22 & \\
\hline
\end{tabular}

A utilização da estatística de Mahalanobis $\left(\mathrm{D}^{2}\right)$, leva em consideração as associação entre as características por meio da matriz de variâncias e covariâncias residuais entre as variáveis (Nunes, 2008). De acordo com Rinderer et al. (1993) a análise discriminante tem sido amplamente empregada em estudos com abelhas do gênero Apis, para separar populações de abelhas africanizadas de populações europeias. Güler et al. (2010) observaram uma alta variabilidade entre as subespécies A. mellifera carnica e A mellifera caucasica utilizando a morfometria geométrica da asa anterior em diferentes regiões ecológicas, demonstrando assim, a eficácia do método para avaliar as diferenças populacionais. Francoy (2007) estudando a variabilidade genético-mofológica em populações Netropicais de Apis mellifera obteve em seus resultados diferença significativa entre as subespécies avaliadas, esse resultado corrobora com o obtido nessa pesquisa.

\section{Conclusão}

Através da morfometria geométrica das asas foi possível verificar o posicionamento racial das abelhas africanizadas do estado do Piauí. Foi possível verificar diferenças na forma das asas das subespécies de Apis mellifera utilizando a morfometria geométrica. Não ocorreu à existência de ecótipos entre as abelhas africanizadas da região litorânea e sertão piauiense.

\section{Agradecimentos}

Agradecemos ao Prof. Dr. Stefan Fuchs, do Institut für Bienenkunde em Oberursel - Alemanha, e ao Prof. Dr. Tiago Maurício Francoy da USP, que gentilmente cedeu as imagens das asas com as referências do padrão de venação das asas anteriores das subespécies Apis mellifera ligustica, Apis mellifera carnica, Apis mellifera melífera e Apis mellifera scutellata. 


\section{Referências bibliográficas}

Arruda, V. M., Alves Jr, V. V., Moraes, M., Chaud Netto, J. \& Suárez, Y. R. (2007). Análise morfológica da glândula de veneno de Apis mellifera L. (Hymenoptera: Apidae) em populações de Mato Grosso do Sul. Neotropical Entomology, 36(2):203-209.

Fonseca, R., Pires, A. V., Lopes, P. S., Torres, R. A. \& Euclydes, R. F. (2000). Estudo da divergência genética entre raças suínas utilizando técnicas de análise multivariada. Arquivo Brasileiro de Medicina Veterinária e Zootecnia, 52(4):403-409.

Francoy, T. M. (2007). Variabilidade genético-morfológica em populações neotropicais de Apis mellifera. PhD, Universidade de São Paulo, Ribeirão Preto, São Paulo, Brasil.

Francoy, T. M. \& Imperatriz-Fonseca, V. L. (2010). A morfometria geométrica de asas e a identificação automática de espécies de abelhas. Oecologia Australis, 14(1):317-321.

Güler, A., Bek, Y. \& Guven, H. (2010). The importance of morphometric geometry on discrimination of Carniolan (Apis mellifera carnica) and Caucasian (A. m. caucasica) honey bee subspecies and in determining their relationship to thrace region bee genotype. Journal of the Kansas Entomological Society, 83(2):154-163.

Nunes, L. A. 2008. Estudo morfológico das populações de Melipona quadrifasciata anthidioides Lepeletier (Hymenoptera: Apidae) na região semi-árida do estado da Bahia. Universidade Federal do Recôncavo da Bahia, Cruz das Almas-BA. 2008. 67 pp.

Nunes, L. A. (2012). Estruturação populacional, variações fenotípicas e estudos morfométricos em Apis mellifera (Hymenoptera: Apidae) no Brasil. PhD, Escola Superior de Luiz de Queiroz, Piracicaba, São Paulo, Brasil.

Nunes, L. A., Araújo, E. D., Marchini, L. C. \& Moreti, A. C. (2012). Variation morphogeometrics of Africanized honey bees (Apis mellifera) in Brazil. Iheringia. Série Zoologia, 102(3):321-326.

Nunes, L. A., Costa, M. d. F. F., Carneiro, P. L. S., Pereira, D. G. \& Waldschmidt, A. M. (2007). Divergência genética em Melipona scutellaris Latreille (Hymenoptera: Apidae) com base em caracteres morfológicos. Bioscience Journal, 23( ):1-9.

Quiroga, C. F. P. (2004). Estrutura genética das populações de abelhas africanizadas (Apis mellifera L.) da Colômbia estimada através de marcadores nucleares e mitocondriais. $\mathrm{PhD}$, Universidade Federal de São Carlos, São Carlos, São Paulo, Brasil.

Rinderer, T. E., Buco, S. M., Rubink, W. L., Daly, H. V., Stelzer, J. A., Riggio, R. M. \& Baptista, F. C. (1993). Morphometric identification of Africanized and European honey bees using large reference populations. Apidologie, 24(6):569-585.

Rohlf, F. J. (2005). tpsDig, digitize landmarks and outlines, version 2.05. New York, USA: Stony Brook.

Souza, D. L., Evangelista-Rodrigues, A., Ribeiro, M. N., Padilla Álvarez, F., Farias, E. S. L. \& Pereira, W. E. (2009). Análises morfométricas entre apis mellifera da mesorregião do sertão paraibano. Archivos de Zootecnia, 58(221):65-71.

Winston, M. L. (1991). The biology of the honey bee: Harvard University Press.

Recebido: 26 de setembro, 2019.

Aprovado: 16 de novembro, 2019.

Publicado: 31 de dezembro,2019.

Licenciamento: Este artigo é publicado na modalidade acesso aberto sob a licença Creative Commons Atribuição 4.0 (CC-BY 4.0), a qual permite uso irrestrito, distribuição, reprodução em qualquer meio, desde que o autor e a fonte sejam devidamente creditados. 